\title{
Recurrent Pyogenic Granuloma - A Clinical Evaluation
}

\author{
Dhamija $\mathbf{M}^{1}$, Singh $\mathbf{P}^{2}$, Dhamija $\mathbf{P}^{3}$
}

\begin{abstract}
The pyogenic granuloma is a localized tumour like overgrowth in the oral cavity caused by reaction to mild irritation. It is considered to be non-neoplastic in nature. Clinically, oral pyogenic granuloma is a smooth or lobulated exophytic lesion manifesting as small, red erythematous papules on a pedunculated or sometimes sessile base, which is usually haemorrhagic. Treatment of pyogenic granuloma consists of conservative surgical excision which is usually curative. There is a relatively high rate of recurrence (about 15\%) after simple excision. This case report describes a recurrent intraoral pyogenic granuloma which was successfully treated with surgical excision and scaling teeth without any significant complications.
\end{abstract}

Keywords: Pyogenic Granuloma, Haemorrhagic, Surgical excision.

${ }^{1}$ Professor and Head

Department of Periodontics

Shree Bankey Bihari Dental College and

Research Centre, Ghaziabad,

Uttar Pradesh, INDIA

${ }^{2}$ Senior Lecturer

Department of Periodontics

BR Ambedkar Dental College and Research Centre,

Patna, Bihar, INDIA

\section{${ }^{3}$ Professor}

Department of Oral Surgery

Shree Bankey Bihari Dental College and

Research Centre, Ghaziabad,

Uttar Pradesh, INDIA

\section{Contact Autbor \\ Dr. Manoj Dhamija drmanojdhamija@gmail.com}

J Oral Health Comm Dent 2013;7(2)132-133

\section{INTRODUCTION}

$\mathrm{P}$ yogenic granuloma $(\mathrm{PG})$ is a rela tively common, soft tissue tumor of the oral cavity that is believed to be reactive and not neoplastic in nature (1, 2). The name pyogenic granuloma is a misnomer since the condition is not associated with pus and does not represent a granuloma histologically (3). It is thought to represent an exuberant tissue response to local irritation or trauma.

Clinically, oral pyogenic granuloma is a smooth or lobulated exophytic lesion manifesting as small, red erythematous papules on a pedunculated or sometimes sessile base, which is usually haemorrhagic. The surface ranges from pink to red to purple, depending on the age of the lesion. It appears as a red mass because it is composed of hyperplasic granulation tissue in which capillaries are prominent (4). Although excisional surgery is the choice of treatment, some other treatment protocols like the use of $\mathrm{Nd}$ :YAG laser, flashed lamped pulsed dye laser, cryosurgery, intralesional injection of ethanol or corticosteroid and sodium tetradecyl sulfate sclerotherapy have been pro$\operatorname{posed}(5)$.

This case report describes a recurrent intraoral PG which was successfully treated with surgical excision and scaling teeth without any significant complications.

\section{CASE REPORT}

An 18-years female patient, reported to the Department of Periodontics, with chief complaint of gingival swelling over maxillary incisors since last 6 month. (Figure 1) History revealed that she had a similar swelling one year earlier which was excised by a local dentist but the lesion again recurred. The lesion bled while brushing. The patient had no systemic complications.

Intraoral examination revealed poor oral hygiene, generalized inflammation of the gingiva and bleeding on probing, especially around maxillary central incisors. During the clinical examination calculus was de-

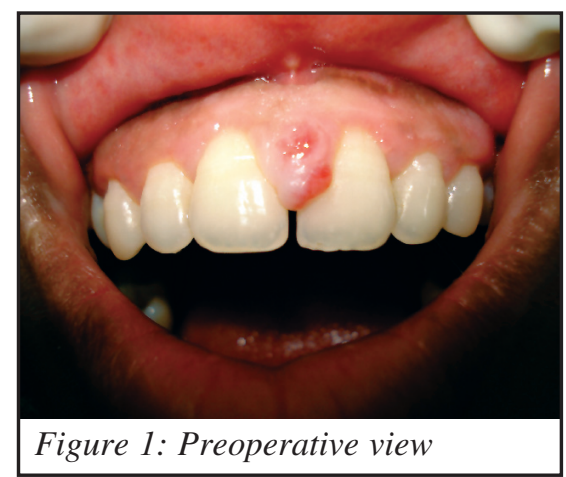




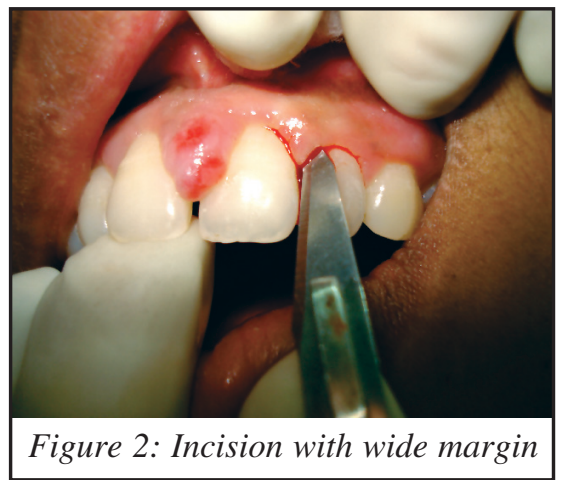

tected under the surface of the mass. Both teeth 11 and 21 responded positively to an electric pulp test. A provisional diagnosis of mild gingivitis associated with a reactive lesion was made.

Surgical excision of the mass was planned. Bilateral infra-orbital nerve block was given. Excisional biopsy of the swelling with a wide margin was performed. (Figure 2-4) The enlarged gingival tissue at the distal and labial aspect was excised. COEpack was placed over the raw surface. (Figure 5-6) The excised mass was sent to laboratory for biopsy. There was uneventful healing after one week. (Figure 7)

Gross tissue examined revealed a single soft tissue bit measuring $1.0 \times 0.7 \mathrm{~mm}$. the histopathogical section showed parakeratinized stratified squamous epithelium with supporting connective tissue. Epithelium was hyperplastic with areas of branching coalesce rete ridges. The overlying epithelium showed hyperchromatic basal cell layer. The underlying connective was fibrocellular with large areas of ossification and showed hyperchromatisa, large endothelial line RBCs capillaries. The overall histopathological features were suggestive of healing pyogenic granuloma with areas of ossification.

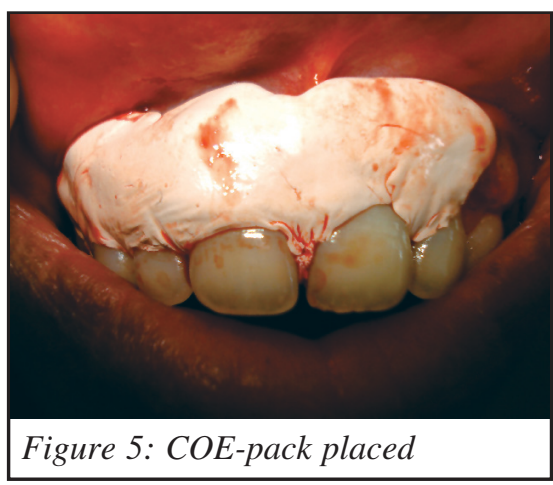

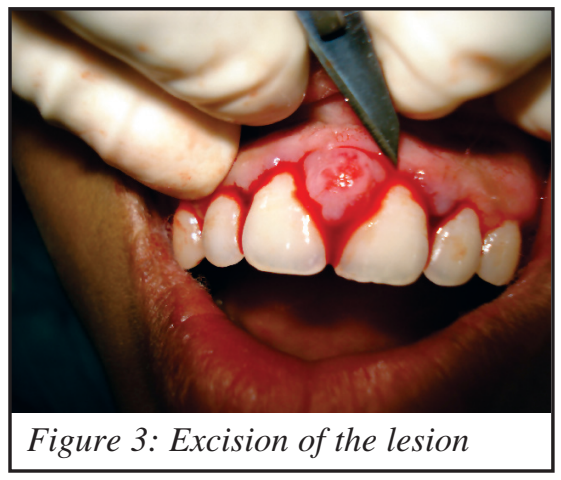

\section{DISCUSSION}

Pyogenic Granuloma is a common, usually solitary, lobulated, benign vascular proliferation of the skin and the mucous membranes, present as hemorrhagic, sessile pedunculated growth. The etiology of the lesion is not known. It is theorized that PG possibly originates as a response of tissues to minor trauma and/or chronic irritation. PG of oral cavity occurs at any age and in all populations without any racial predilection (6).

The typical clinical presentations of PG are a small, deep red to reddish-purple lesion occurring on the gingiva, which is either sessile or pedunculated. The surface may be smooth, lobulated or occasionally ulcerated and shows a tendency for hemorrhage either spontaneously or upon slight trauma. The lesion is painless and soft in consistency, although the older lesions tend to become more collagenized and firm. The size of the lesion usually ranges between $0.5 \mathrm{~cm}$ and $2 \mathrm{~cm}$, and hey grows at an alarming rate reaching that size within 4 to 7 days.

All clinically suspected pyogenic granulomas must be biopsied to rule out more serious conditions as mentioned previously. The histopathological picture of the extra

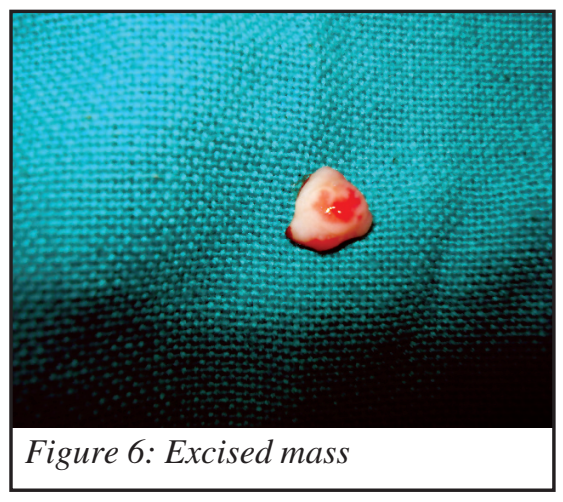

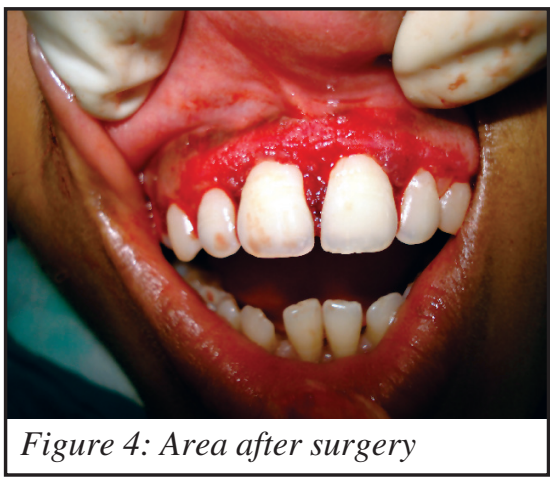

gingival pyogenic granuloma is quit similar to the ones occurring on the gingival. Microscopically, it consists of many dilated blood vessels in a loose edematous connective tissue stroma. There is typically a dense acute inflammatory infiltration but this may be scanty or absent.

Treatment of pyogenic granuloma consists of conservative surgical excision which is usually curative. There is a relatively high rate of recurrence (about 15\%) after simple excision (7).

\section{REFERENCES}

1. Amirchaghmaghi $M$, Falaki $F$, Mohtasham $N$, Mozafri PM. Extragingival pyogenic granuloma: a case report. Case Journal 2008;1:371.

2. Nevile BW, Damm DD, Allen CM, Bouquot JE. Oral and maxillofacial pathology Second edition. WB Saunders Co. 2004: pp 444-49.

3. Shafer WG, Hine MK, Levy BM. A textbook of oral pathology, $4^{\text {th }} \mathrm{ed}$, WB Saunders, Philadelphia, 1983:pp359-60.

4. Vossoughi R, Takei H. External cervical resorption associated with traumatic occlusion and pyogenic granuloma. JADC2007;73(7):62527.

5. Jafarzadeh $\mathrm{H}$, Sanatkhani $\mathrm{M}$, Mohtasham $\mathrm{N}$. Oral pyogenic granuloma: a review. J Oral Sci 2006;48(4):167-75.

6. Patil K, Mahima VG, Lahari K. Extragingival pyogenic granuloma. IJDR 2006;17(4):199-202.

7. Tolentono ES, Tolentino LS. Recurrent intraoral pyogenic granuloma: case report. Odontolgia Clin Clientif 2009;8(3): 266-67.

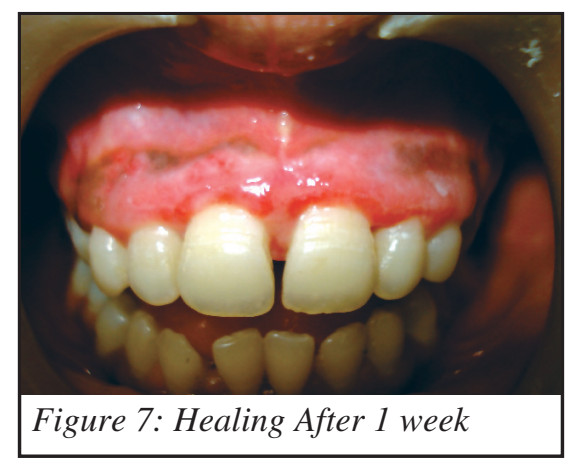

